\title{
Intermédialités
}

Histoire et théorie des arts, des lettres et des techniques

Intermediality

History and Theory of the Arts, Literature and Technologies

\section{Marx, Wittgenstein et l'amante du mage : remarques sur la disparition, l'évidence et le pouvoir}

\section{Valeria Wagner}

Numéro 10, automne 2007

Disparaître

Disappearing

URI : https://id.erudit.org/iderudit/1005550ar

DOI : https://doi.org/10.7202/1005550ar

Aller au sommaire du numéro

Éditeur(s)

Centre de recherche sur l'intermédialité

ISSN

1705-8546 (imprimé)

1920-3136 (numérique)

Découvrir la revue

Citer cet article

Wagner, V. (2007). Marx, Wittgenstein et l'amante du mage : remarques sur la disparition, l'évidence et le pouvoir. Intermédialités / Intermediality, (10), 17-32. https://doi.org/10.7202/1005550ar
Résumé de l'article

Un mage fait disparaître la tour Eiffel devant une foule étonnée, mais son amante résiste à son charme, et s'inquiète de l'usage que l'on pourrait faire de ce pouvoir singulier. Comment, et pourquoi, l'amante voit-elle, alors que les autres ne voient pas? Comment, en définitive, résister comme elle au pouvoir trompe-l'oeil, délocalisé et trans-personnel, qui agit par la perception plutôt que par la force, en changeant les contours du paraître? Ces questions sont poursuivies d'après certaines lignes de réflexion parallèles de Marx et de Wittgenstein, qui s'étendent sur les dangers que l'amante du mage pressent dans cet art de la disparition. Les deux philosophes se posent comme les témoins qui " voient » à travers les actes d'illusionnisme qui déforment, pour l'un, notre perception des relations sociales et de leur lien aux rapports de production, et pour l'autre, notre compréhension des formes d'expression et de leur imbrication dans les formes de vie qui leur donnent sens. Et, comme l'amante du mage, ils « voient " dans ces disparitions et apparitions des enjeux considérables, autant pour le " public » que pour les acteurs de ces spectacles quotidiens, puisqu'ils impliquent le transfert du pouvoir des agents à des instances désincarnées ou mystérieusement animées. 


\title{
Marx, Wittgenstein et l'amante du mage: remarques sur la disparition, l'évidence et le pouvoir
}

\author{
Valeria Wagner
}

iguel Quiroga ${ }^{1}$, protagoniste désœuvré et sans argent du film El acto en cuestión (L'acte en question², Alejandro Agresti, Argentine, 1993), a la curieuse habitude de voler au hasard des livres dans des librairies d'occasion, qu'il lit ensuite minutieusement d'un bout à l'autre. Selon une amante perspicace, il suit ainsi, inconsciemment, le conseil de son père, qui lui aurait assuré qu'il trouverait dans les livres tout ce dont il aurait besoin dans la vie. Cette foi parentale dans l'utilité des livres se confirme de manière inattendue lorsque Quiroga s'empare, toujours par hasard, du texte qui changera sa vie: Magie et occultisme. Il y apprend un tour de disparition si réussi qu'aucune médiation corporelle ne semble y intervenir, aucune ruse n'y est détectable, et ce même à ses propres yeux. Le premier objet qu'il fait disparaître est, d'ailleurs, un télescope, signe de l'effet que sa magie aura sur les regards.

Engagé d'abord dans un cirque, son spectacle est un succès. Bientôt Quiroga parcourt les quatre continents, devenant riche et célèbre. Mais le pouvoir singulier sur lequel repose désormais son existence le tracasse. D’une part, il est d'autant plus affecté par le mystère des disparitions qu'il ne sait pas comment fonctionne sa propre ruse - comment les choses et les êtres disparaissent-ils? Qu'est-ce qui se passe? D'autre part, il vit dans la crainte que d'autres exemplaires du livre de magie (il a déjà brûlé le «sien») existent et soient lus, et que «son»

1. Le nom est un composite de Miguel de Cervantes, auteur du Don Quichotte, et de Horacio Quiroga, auteur argentin connu pour ses contes fantastiques.

2. Mes remerciements à Andreas Fontana, qui m’a procuré une copie du film, très difficile à trouver. 
acte devienne public 3 . Il décide alors d'arrêter ses spectacles afin de retrouver son calme, mais accepte de se produire pour une dernière fois à Paris, où il fait disparaitre la tour Eiffel devant les yeux étonnés d'une foule. Là, au sommet de sa gloire, ses doutes et ses craintes se concrétisent lorsque son amante du moment, Sylvie, reste insensible au charme de sa magie. Elle ne voit pas de disparition, elle continue à voir ce que les autres ne voient plus. Sûre de sa vision, elle est effrayée par la crédulité du public, craignant que le pouvoir que son amant met au service du divertissement ne soit utilisé par d'autres à des fins bien plus nocive4.

À la suite de ce questionnement, des fondements et de la sphère d'application de son pouvoir, le mage est hanté par la peur que son amante ne révèle ce qu'elle a vu (ou n'a pas vu), et il en fait sa prisonnière. S'ensuit une relation sadomasochiste complexe qui ne prend fin que lorsque son gérant, ex-directeur du cirque, lui apprend que c'est lui qui avait sorti de la circulation le livre Magie et occultisme, et qu'il va désormais en faire réimprimer des milliers d'exemplaires. Accusé alors de violer les droits d'auteur, Quiroga redevient face à la loi le simple voleur qu'il était au début. Pouvoir, fortune, gloire et amante disparaissent. À la fin du film, l'ex-mage est réduit à travailler dans l'atelier de jouets et de poupées d'un vieil ami, où il continue en quelque sorte à exercer un métier d'illusionniste, cette fois sans tour de magie interposé.

Le film pourrait être lu comme une fable moralisante sur la nature illusoire et éphémère du pouvoir et de la gloire, s'il ne laissait pas ouverte la question de la nature de l'acte de disparition. Pour une mentalité contemporaine, la disparition physique est relative à un point de vue et peut en principe être expliquée scientifiquement et ramenée à une modalité du paraître. La disparition,

3. Il fouille dans les bibliothèques, soupçonne tous les lecteurs de chercher à découvrir «son » acte, dénigre les «intellectuels», qui croiraient que la vérité se trouve dans les livres.

4. Pour un public renseigné, les tours de disparition du mage ne manquent pas de rappeler ceux ayant rendu mondialement célèbres les militaires argentins pendant la dictature de 1976-83, pendant laquelle quelques 30000 personnes ont été séquestrées, enfermées dans des camps de détention clandestins, et secrètement assassinées. Les liens entre les tours de magie et la disparition forcée des personnes ne sont d'ailleurs pas seulement circonstanciels. En effet, bien qu'ils s'accomplissent sur des modes fondamentalement différents - l'un ludique, l'autre, d'un sérieux dévastateur - tant les actes de disparition de lapins et de tours, comme ceux de personnes et de groupes, doivent leur capacité d'émerveiller ou de terroriser à l'occultation du pouvoir et des moyens qui font disparaître. Cependant, je ne vais pas développer ici la disparition dans le cadre du terrorisme d'État, me concentrant plutôt sur les processus de délocalisation et d'attribution du pouvoir que la figure de la disparition met en évidence. 
en définitive, "paraît», mais elle ne peut pas «être »5. Ceci est d'autant plus vrai lorsqu'il s'agit de mises en scène de la disparition, comme dans le cas de tours de magie. Pour réussir son acte de disparition, le mage doit cacher tout lien matériel entre l'événement et lui-même, et créer l'illusion qu'il est capable d'agir à distance sur la matière sans autre intermédiaire physique que les mots. Il doit donner l'impression que le pouvoir d'action réside dans ses mots ou dans les gestes esquissés dans l'air. Mais dès que l'opération de disparition peut être liée à des moyens et des agents spécifiques, l'acte du mage n’apparaît plus comme la manifestation d'un pouvoir spirituel ou désincarné et redevient la démonstration d'un savoir-faire comme un autre, aussi complexe et admirable soit-il. Dans ce sens, dans le film d'Agresti, la réédition de Magie et occultisme devrait, en principe, suffire à dissiper l'effet mystificateur des disparitions en établissant un lien clair entre «l'acte en question » et la personne physique du mage qui le met en œuvre, et à révéler ainsi le trompe-l'œil sur lequel se fonde son pouvoir.

Pour la loi, c'est en effet suffisant: le livre tient lieu du chaînon manquant qui révèle la ruse et le crime du mage (le mépris des droits d'auteur). Cependant, ni l'inculpation ni la révélation de la ruse n'élucident complètement le mystère de «l'acte en question ». D'une part, nous le savons, le mage lui-même ne comprend pas comment il réussit son tour - il peut exécuter l'opération, en suivant les instructions du livre, sans pour autant la maîtriser et sans connaître les raisons de son succès. Dans un sens, il est bien l'agent responsable de l'acte, mais dans un autre, il le subit, jusqu'au point qu'il semble n'être que le moyen à travers lequel des pouvoirs invisibles se manifestent dans le monde physique. En même temps, comme nous l'apprenons à la toute fin du film, Quiroga semble être le seul à réussir ces tours de disparition, et ce malgré la publication du livre d'où il a tiré son savoir faire. Le rôle du livre devient ainsi incertain, puisque le lien entre instruction et exécution reste aléatoire. Finalement, la clairvoyance de Sylvie, la deuxième amante, démontre que le livre n'est pas nécessaire pour percer le tour de disparition. En effet, tandis que le mage, qui l'a lu, voit les objets disparaître, elle, sans l'avoir lu, ne voit pas de disparition, et peut même préserver avec sa vision l'apparence des objets qui échappent à la perception de son amant et du

5. En d'autres mots, elle n'existe pas dans l'absolu: les peuples et espèces disparus laissent des traces et des questions qui, bien qu'en suspens, peuvent en principe avoir des réponses; des morts, dont on dit qu'ils «disparaissent», restent les corps ou les histoires, sauf des cas spéciaux où manquent les dépouilles et les témoins de la mort (le cas des disparus en Argentine, des cas d'expéditions, les disparitions volontaires, etc.). Mais le mystère de la disparition est, dans ces cas, circonstanciel. 


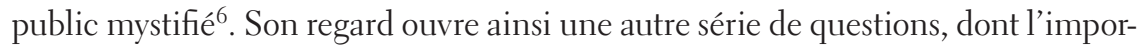
tance déplace toute conclusion moralisante : comment et pourquoi l'amante voitelle, alors que les autres ne voient pas? Comment son regard résiste-t-il aux tours de disparition? Comment, en définitive, résister comme elle au pouvoir trompel'œil, délocalisé et trans-personnel, qui agit par la perception plutôt que par la force, en changeant les contours du paraître?

Ces questions dépassent le cadre de Lacte en question, comme nous allons voir, en suivant certaines lignes de réflexion parallèles de Marx et de Wittgenstein, qui s'étendent sur les dangers que l'amante du mage pressent dans cet art de la disparition7 ${ }^{7}$ Les deux philosophes se posent, chacun à leur manière, comme les témoins qui «voient» à travers les actes d'illusionnisme qui déforment, pour l'un, notre perception des relations sociales et de leur lien aux rapports de production, et pour l'autre, notre compréhension des formes d'expression et de leur imbrication dans les formes de vie qui leur donnent sens. Comme l'amante du mage, ils voient encore ce que les autres ne voient plus, identifiant de surcroît les mirages qui surgissent à la place. Et comme elle, ils voient dans ces disparitions et apparitions des enjeux considérables, autant pour le «public» que pour les acteurs de ces spectacles quotidiens, puisqu'ils impliquent le transfert du pouvoir des agents à des instances désincarnées ou mystérieusement animées. Ainsi Marx constate que les acteurs sociaux, loin de gérer leurs rapports de production, se retrouvent sous leur emprise, tandis que Wittgenstein montre comment les usagers du langage succombent au charme des mots et des expressions qu'ils utilisent. Comme dans le film d'Agresti, «les tours de magie» qui asservissent les acteurs sociaux et enchantent les usagers du langage dépassent les mages et criminels spécifiques, qui sont plutôt présentés, nous le verrons, comme les médiateurs des «tours» qu'ils subissent, lesquels seraient autant l'effet inévitable des conditions de la société et du langage que de la manière dont on se méprend sur elles. Sans recourir au modèle du sujet autonome maître de ses actes et de sa signification - d'ailleurs les «enchantements » auxquels il est sujet seraient

6. Notons à cet égard que le film autorise tant le regard démystificateur de l'amante que celui, mystifié, du public et du mage : la caméra montre la tour lorsqu'elle tient lieu du regard de la première, et montre son absence lorsqu'elle se place dans la perspective du deuxième.

7. Ce film me semble particulièrement important en ce qu'il propose une réflexion sur le pouvoir et la disparition dissociée des événements historiques traumatiques de la dictature militaire. Le recours au mage, notamment, remet la disparition dans le contexte à la fois familier et exceptionnel du spectacle et du divertissement, introduisant la distance ludique nécessaire pour repenser le pouvoir et la disparition en dehors du contexte du terrorisme d'État. 
la preuve que ce modèle est lui-même un «mirage» - Marx et Wittgenstein entreprennent de ramener le pouvoir au sein des relations sociales et linguistiques où il opère, en attirant notre attention sur les disparitions et apparitions qui marquent les déplacements du pouvoir.

Dans la première section du Capital $^{8}$, Marx reprend le diagnostic de la société de son temps qu'il développe tout au long de ses écrits. «La vie sociale», affirme-t-il, est voilée d'un «nuage mystique» (C, p. 74), notamment parce que son assise sur les rapports de production n'est pas immédiatement perceptible. Ces rapports sont enfouis dans la série de «disparitions » qui accompagnent l'abstraction de la valeur d'usage du produit du travail et la transformation de ce dernier en marchandise ayant de la valeur d'échange:

Ce n'est plus [...] un objet utile quelconque; [...] ce n'est pas non plus le produit [...] de n’importe quel travail déterminé. Avec les caractères utiles particuliers des produits du travail disparaissent en même temps, et le caractère utile des travaux qui y sont contenus, et les formes concrètes diverses qui distinguent une espèce de travail d'une autre espèce. (C, p. 43)

C'est par ce dépouillement que les produits de travail sont «[m]étamorphosés en sublimés identiques, échantillons du même travail indistinct» (c, p. 43), acquérant en même temps de la valeur d'échange et la «réalité fantomatique » (c, p. 43) propre aux marchandises:

Une marchandise paraît au premier coup d'œil quelque chose de trivial et qui se comprend de soi-même. Notre analyse a montré au contraire que c'est une chose très complexe, pleine de subtilités métaphysiques et d'arguties théologiques. En tant que valeur d'usage, il n'y a en elle rien de mystérieux, [...] la table reste bois, une chose ordinaire et qui tombe sous les sens. Mais dès qu'elle se présente comme marchandise, c'est une tout autre affaire. A la fois saisissable et insaisissable, il ne lui suffit pas de poser ses pieds sur le sol; elle se dresse, pour ainsi dire, sur sa tête de bois en face des autres marchandises et se livre à des caprices plus bizarres que si elle se mettait à danser. (c, p. 68)

Outre leur apparence superficielle, les marchandises gardent peu de traces du travail qui les a produites; elles semblent même être animées de vie propre, jusqu'au point que leurs relations et leurs projets9 semblent déterminer le

8. Karl Marx, Le Capital. Livre I. Sections I à IV [1867], trad. Joseph Roy, Paris, Éditions Flammarion, 1985. Désormais, les références à cet ouvrage seront indiquées par le sigle « C », suivi de la page et placées entre parenthèse dans le corps du texte.

9. Marx parle souvent du langage, des pensées et des intentions des marchandises, au moyen desquelles il personnifie la manière dont on les considère et on les traite, et met en évidence l'animisme sous-jacent la pensée et le comportement économique. Voir, 
comportement social des humains. Aux yeux des hommes pris dans l'engrenage de la production marchande, «leur propre mouvement social prend [...] la forme d'un mouvement des choses [...]» (C, p. 71), une fantasmagorie qui exprime à la fois le fait et l'illusion que «la production et ses rapports régissent l'homme au lieu d'être régis par lui » (C, p. 75).

Il s'agit d'une illusion, parce que la production reste toujours concrètement entre les mains des acteurs sociaux; ce sont eux qui produisent des marchandises avec leur travail, et les «animent» les faisant circuler; c'est aussi eux qui font fonctionner le mystérieux système monétaire, accumulent du capital, et s'exploitent les uns les autres. Mais en même temps, la projection du pouvoir des acteurs sociaux sur les produits (aliénés) de leur travail exprime une certaine réalité, à savoir, que la société n'est pas constituée d'hommes librement associés, «agissant consciemment et maîtres de leur propre mouvement social» (c, p. 74-75). Pour autant qu'elle soit envisageable, l'avènement d'une telle société ne dépend pas pour Marx d'une volonté ou conscience sociale; il n'est possible qu'avec la réalisation de certaines conditions matérielles (touchant notamment les moyens de production), qui ne "peuvent être elles-mêmes le produit que d'un long et douloureux développement» (c, p. 74-75). Même lorsqu'une société a «découvert» ou «dévoilé » la loi naturelle qui préside à son mouvement (c, p. 37), avertit Marx dans sa préface à la première édition du Capital (1867), elle ne peut qu'aspirer à réduire ses souffrances et à accélérer le processus de changement pour qu'il aboutisse. Ces propos nuancent la fameuse $\mathrm{XI}^{\mathrm{e}}$ thèse sur Feuerbach, où Marx reproche à ses confrères philosophes de n'avoir fait «qu'interpréter le monde de diverses manières », alors qu'il s'agit aussi «de le transformer ${ }^{10}$ ». La «loi naturelle» du devenir de la société ne pouvant être ni changée ni dépassée, la «transformation » en question porte plutôt sur «la forme » que prend le monde (social) aux yeux des philosophes et des acteurs sociaux. Il s'agit alors d'en « dévoiler» le fonctionnement, de redonner visibilité aux rapports sociaux et aux rapports sousjacents de production qui déterminent son humanité, et d'intervenir, en somme, dans la construction de sa factualité et de ses évidences. Car, nous l'avons vu,

par exemple, la fin de 1.4, la partie sur le fétichisme: «Les marchandises diraient, si elles pouvaient parler: "[...] Nous ne nous envisageons les unes les autres que comme valeurs d'échange”. Ne croirait-on pas que l'économiste emprunte ses paroles à l'âme même de la marchandise, quand il dit: "la valeur (valeur d'échange) est une propriété des choses [...]"? » (c, p. 75-76).

10. Karl Marx, «Ad Feuerbach (Thèses sur Feuerbach)» [1845], dans Karl Marx, Philosophie, Maximilien Rubel (éd.), trad. Maximilien Rubel et Louis Janover, Paris, Éditions Gallimard, coll. «Folio essais », 1982, p. 232-36. 
les apparences sont trompeuses - ce qui semble se donner en toute simplicité, comme la marchandise, cache des degrés de complexité insoupçonnés, tandis que «quelque chose de très simple», comme le rapport entre la «forme valeur» et «la forme monnaie», a laissé perplexe des générations d'illustres économistes (C, p. 36).

Malgré des orientations différentes - philosophie du langage, philosophie économique et politique - et parfois même apparemment opposées, la tâche que Wittgenstein assigne à la philosophie dans ses Recherches philosophiques ${ }^{11}$ est étonnamment semblable au projet de transformation du monde de Marx. Dans une de ses remarques les plus célèbres, Wittgenstein rappelle à ses confrères que la philosophie «laisse toute chose en l'état»; elle «se contente de placer toute chose devant nous, sans rien expliquer ni déduire» (RP, $\mathbb{1 2 6}$ ). Mais pour ce faire, elle doit combattre «l'ensorcellement de notre entendement par les ressources de notre langage» (RP, $\left.\int 109\right)$ - briser le charme à la fois hypnotique et incantatoire des certitudes et des évidences ${ }^{12}$, démonter les «formes d'expression» qui «nous lancent à la chasse aux chimères » (RP, $\left.\int 94\right)$, ramener les «superstitions » aux «illusions grammaticales» qui les génèrent (RP, $\mathbb{S} 110)$. Il s'agit, en définitive, de mettre en évidence les tours de magie du langage, qui font miroiter, par exemple, l'essence des «choses » nommées par les mots «je », « objet», ou "proposition» (RP, $\mathbb{S} 116)$, ou qui créent l'illusion que des actes aussi courants comme, par exemple, «montrer la forme» $(\mathrm{RP}, \mathbb{S} 6)$, sont réalisés par des entités spirituelles, telles «la pensée », ou «l'intention». Ce dernier cas a valeur d'exemple générique :

Nous faisons ici ce que nous faisons dans quantité de cas analogues: étant donné que nous ne pouvons pas indiquer une action corporelle que nous appellerions «montrer la forme» (par opposition à la couleur, par exemple), nous disons qu'il y a une activité de l'esprit qui correspond à ces mots.

Là où notre langage nous suggère qu'il y a un corps et qu'il n'y en a pas, nous aimerions dire qu'il y a un esprit. (RP, $\left.\mathbb{S}_{3} 6\right)$

11. Ludwig Wittgenstein, Recherches philosophiques [1953], trad. Françoise Dastur, Maurice Élie, Jean-Luc Gautero, Dominique Janicaud, Élisabeth Rigal, Paris, Éditions Gallimard, coll. «Bibliothèque de philosophie», 2005. Désormais, les références à cet ouvrage seront indiquées par le sigle «RP », suivi du numéro de la remarque (\$) et placées entre parenthèse dans le corps du texte.

12. Par exemple: «Je me dis et je me répète à moi-même: il en est pourtant ainsi. J'ai l'impression que si je pouvais ajuster avec précision mon regard sur ce fait de façon à l'amener au foyer de ma vision, je devrais saisir l'essence de la chose en question. » (RP, \113) Ou encore: «Une image nous tenait captifs. Et nous ne pouvions lui échapper, car elle se trouvait dans notre langage qui semblait nous la répéter inexorablement» (RP, $\$ 115)$. 
Nous sommes devant une transposition analogue à celle décrite par Marx, lorsqu'il remarque que les hommes attribuent aux choses ce qui leur revient en tant qu'êtres sociaux. Ici, les locuteurs, «oubliant» leur rapport au langage, traitent les mots comme si c'étaient des choses et que celles-ci dictaient, à leur tour, l'usage des mots. On suppose que si l'expression «montrer la forme » existe, il y a soit une entité idéale «forme» détachable de l'objet en question, ou bien un acte spirituel bien distinct, et différent de «montrer la couleur». «Nous prédiquons de la chose ce qui réside dans l'ordre de la représentation» (RP, $\left.\int 104\right)$, de sorte que cette dernière paraît refléter l'ordre des choses. Il semble alors que les choses s'auto-(re)présentent et produisent elles-mêmes le langage qui, outre porter en lui la connaissance des choses en-soi, doit être utilisé de manière univoque, selon des principes «absolus».

Le résultat d'une telle transposition est une sorte d'affranchissement du langage, qui produit un monde à sa guise et entraîne une perte de pouvoir effectif de ses usagers. Ceux-ci ont alors l'impression, soit de ne pas être à la hauteur du langage, soit que ce dernier n'est pas à la hauteur de la réalité, tant et si bien que, malgré les preuves quotidiennes du contraire, aucune médiation entre le langage et la réalité ne semble possible. Or, selon la manière dont les opérations langagières sont comprises, ces impressions semblent fondées : certaines formes d'expressions peuvent effectivement être appréhendées de façon telle qu'elles semblent conçues «pour un Dieu qui sait tout ce que nous ne pouvons pas savoir» (RP, $\left.\mathbb{\int} 426\right)^{13}$, d'autres suggèrent l'existence d'entités que le langage ne saurait appréhender. Pour dissiper ces impressions, il suffit de rester «aux choses de la pensée quotidienne », résister à la tentation de croire «que nous avons à décrire d'extrêmes subtilités que nous ne pourrions pas décrire avec les moyens dont nous disposons » (RP, $\left.\mathbb{\int} 106\right)$, et veiller à « ne pas présenter la situation comme s'il y avait quelque chose que l'on ne pouvait pas faire» $\left(\mathrm{RP}, \mathbb{\int} 374\right)^{14}$. C'est donc dans l'optique d'échapper à l'emprise paralysante des mots que Wittgenstein entend reconduire «les mots de leur usage métaphysique à leur usage quotidien » (RP,

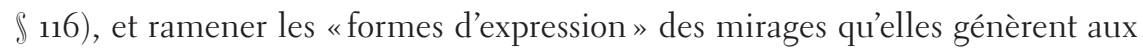
«formes de vie» où elles s'inscrivent.

13. Elles sont alors comparables à «des habits de cérémonie que nous revêtons sans doute, mais dont nous ne pouvons pas faire grande chose, car nous sommes privés du véritable pouvoir qui donnerait un sens et une fonction à cet habit» (RP, $\mathbb{4} 426)$.

14. Ici, il s'agit, comme souvent ailleurs, de descriptions: «Comme s'il y avait effectivement un objet dont je tirerais la description, mais que je serais incapable de montrer à quiconque» $\left(\mathrm{RP}, \mathbb{\int} 374\right)$. 
Le projet de «transformation du monde» de Marx est ainsi comparable à celui de "préservation de l'état des choses» de Wittgenstein en ceci que, dans les deux cas, il y a des fausses évidences à démonter afin de découvrir, ou simplement remarquer, les relations sociales - les rapports de production, l'usage du langage (le rapport productif au langage) - ombragées par les mirages métaphysiques. Soulignons que ni Marx ni Wittgenstein ne présupposent pour autant des évidences «ultimes»: pour le premier tout ce qui semble être simplement donné l'est historiquement, et n'est donc pas immédiatement visible; pour le deuxième, les évidences sur lesquelles reposent les certitudes et les questionnements sont aussi celles que l'on ne voit plus:

Les aspects des choses les plus importants pour nous sont cachés du fait de leur simplicité et de leur banalité. (On peut ne pas remarquer quelque chose - parce qu'on l'a toujours sous les yeux.) Les véritables fondements de sa recherche ne frappent pas du tout l'attention d'un homme. À moins qu'ils ne l'aient frappée à un moment donné. - Ce qui signifie que ce qu'il y a de plus frappant et de plus fort ne frappe plus notre attention, une fois qu'on l'a vu'15. (RP, \$129)

Ici, c'est la visibilité même des aspects importants des choses qui les fait disparaître sous nos yeux du fait de leur simplicité et de leur banalité. Ces aspects fondent la perception et la formulation de ce qui est complexe ou surprenant, passant eux-mêmes inaperçus. Wittgenstein ne décrit pas ce phénomène avec regret; il semble inévitable que questions et certitudes reposent sur un degré d'inattention au banal et au simple. Mais les inattentions qui intéressent tant Marx que Wittgenstein sont l'effet de distractions et diversions spécifiques, liées notamment à l'illusion que les choses s'animent ou aux dédoublements que génère la fétichisation - pour emprunter la terminologie marxienne - des marchandises et des mots et des formes d'expression. Dans un cas comme dans l'autre, la fétichisation - l'attribution de pouvoirs «magiques» aux choses inanimées - est elle-même liée à la méprise de la notion de valeur des choses (leur valeur d'échange) et des mots (leur signification): ainsi les économistes que Marx cite considèrent la valeur comme un attribut des marchandises, comme si la valeur d'échange était une de leurs composantes chimiques (c, p. 76), et un des interlocuteurs imaginaires de Wittgenstein agit (parle) « [...] comme si

15. Pour clarifier le sens de "À moins qu'ils ne l'aient frappé à un moment donné», voir la traduction anglaise de G. E. M. Anscombe (Philosophical Investigations, Oxford, Blackwell, 1989 [1953]): «Unless that fact [that the real foundations of his enquiry do not strike a man at all] has at some time struck him ». Ainsi, l'homme peut seulement remarquer qu'il ne remarque pas les fondements de sa recherche. 
la signification était un halo que le mot portait avec lui et transportait dans tous ses emplois » (RP, $\left.\int 117\right)$. Ni essence, ni substance, ni esprit, soutiennent Marx et Wittgenstein: pour comprendre la valeur des marchandises et la signification des mots, il est nécessaire de les ramener, respectivement, à la valeur d'usage et à l'usage quotidien, c'est-à-dire aux situations où le rapport entre la valeur et les relations sociales est aisément reconnaissable, où il ne disparaît pas avec la transformation métaphysique des produits et des mots.

Le rapport entre la signification des mots et des expressions et leur usage - dans des circonstances particulières, des jeux de langage spécifiques, selon des critères correspondant à des «formes de vie» - est aujourd'hui un lieu commun, bien que, dans la pratique, les mots et les expressions continuent à être essentialisés. C'est le cas, par exemple, de «la conscience», que la plupart des usagers du langage croient posséder inconditionnellement: «[...] si je dis, "J'ai une conscience", à qui à proprement parler est-ce que je donne une information? Dans quel but me dis-je cela, et comment les autres peuvent-ils me comprendre?» (RP, $\left.\int 416\right)$. Si nous continuons, malgré nous, à penser «la signification comme une chose du même genre que le mot, et néanmoins différente de lui1 ${ }^{16} »\left(\mathrm{RP}, \mathbb{\int} 120\right)$, c'est que le langage fonctionne avec des comparaisons et des analogies qui produisent des fausses apparences: la même forme grammaticale est utilisée pour «conscience» que pour «table» (RP, $\mathbb{S} 112)$. À l'usage, ces «fausses apparences» ne nous trompent pas; nous ne guettons pas notre conscience. Ce n'est que lorsque nous nous concentrons sur le mot isolé que nous risquons de tomber dans le piège métaphysique, parce que, bien que l'usage «marque» les mots, les règles d'usage renvoyant aux «formes de vie » spécifiques n'y sont pas inscrites. Wittgenstein dira de ce genre de cas, où les mots et les expressions sont considérés en dehors de leurs différents usages possibles, que «le langage tourne à vide» - il est comme une machine ou un moteur à l'arrêt, il ne «travaille » pas ${ }^{17}$ $\left(\mathrm{RP}, \mathbb{\int} 132\right)$. C'est alors que surgissent les «problèmes philosophiques» $\left(\mathrm{RP}, \mathbb{\int} 38\right)$ et les «confusions» (RP, $\mathbb{S} 132)$ qui occupent Wittgenstein, lesquelles peuvent se clarifier en remettant le langage « en marche », ou «au travail » dans la pratique quotidienne $^{18}$. D'où le besoin de connaître l'emploi différencié du langage : « [...]

16. «On dit: l'important n'est pas le mot, mais sa signification; et on pense alors la signification comme une chose du même genre que le mot, et néanmoins différente de lui. Ici le mot, et là sa signification. L'argent, et la vache que l'on peut acheter avec. (Mais d'un autre côté: L'argent, et son utilité.)» (RP, \120)

17. Dans la traduction anglaise, le langage « is like an engine idling».

18. «Car les problèmes philosophiques surgissent lorsque le langage est en roue libre» $(\mathrm{RP}, \mathbb{S} 8)$, ou bien, «For philosophical problems arise when language goes on holiday». 
nous mettrons constamment en évidence des différences que les formes habituelles de notre langage nous poussent à négliger». (RP, $\left.\int 132\right)$.

Notons l'affinité de ces propos avec ceux de Marx sur la difficulté d'appréhender la marchandise. Cette dernière est «à première vue » tout à fait comparable au produit du travail considéré dans sa valeur d'usage : la table-objet d'utilité et la table-marchandise se présentent de la même manière à la perception. Mais cette ressemblance, nous avons vu, est trompeuse pour Marx, car son évidence empêche de remarquer une différence fondamentale, à savoir, qu'en tant que marchandise la table a de la valeur sociale non pas pour ce qu'elle présente - ses propriétés physiques, son utilité —, mais pour la possibilité d'être échangée contre une autre marchandise ou contre l'équivalent universel qu'est l'argent qu'elle représente. Ainsi, Marx identifie, dans tout produit soumis aux règles de l'échange, la production fantasmatique d'une «forme équivalente » qui le rendra échangeable:

Quand on exprime la valeur de la toile dans l'habit, l'utilité du travail du tailleur ne consiste pas en ce qu'il fait des habits et, selon le proverbe allemand, des hommes, mais en ce qu'il produit un corps, transparent de valeur, échantillon d'un travail qui ne se distingue en rien du travail réalisé dans la valeur de la toile. Pour pouvoir s'incorporer dans un tel miroir de valeur, il faut que le travail du tailleur ne reflète lui-même rien que sa propriété de travail humain. (c, p. 58)

Lorsque nous avons affaire à une table, par exemple, au cours d'une opération d'échange, ou circulant dans le marché, nous pouvons penser n'avoir à faire qu'à une table, mais c'est avec son «corps, transparent de valeur » et «échantillon de travail» abstrait que nous nous affairons. Ce corps, expression de la valeur du travail indifférencié, subit une série de «métamorphoses» (c, p. 61) que Marx suit en détail, jusqu’à atteindre son expression à la fois la plus abstraite et la plus concrète dans l'argent (la forme monnaie, le prix), qui remplace toute marchandise et rend les produits du travail parfaitement et quantitativement commensurables. Or, la valeur d'échange est bien ce qui permet la mobilité des produits du travail ainsi que la «conversion» du travail privé des individus en travail social, rassemblant les forces de production qui semblent divisées dans le même mouvement social qu'est, au fond, le marché. Le problème surgit lorsque la réalité fantasmatique de cette valeur d'échange n'est pas reconnue, et lorsque la valeur est attribuée aux produits spécifiques, alors même qu'elle nie toute leur spécificité et avec elle les liens entre le produit et le travail, et par extension, le travailleur. La perception des marchandises comme des objets ou des choses «comme les autres» implique ainsi l'oubli du travail et des travailleurs qui les produisent. Si Wittgenstein doit rappeler constamment à ses interlocuteurs que le 
langage ne produit pas du sens tout seul et que c'est bien à ses usagers de le faire «travailler», Marx rappelle sans cesse aux acteurs que la valeur, soit-elle d'usage ou d'échange, est produite par leur travail (le propre, celui des autres) ${ }^{19}$.

Lorsque la table-marchandise est considérée comme simple table alors qu'elle est traitée comme marchandise, la situation est analogue à celle du langage «qui tourne à vide» ou qui est comme un moteur à l'arrêt, semant la confusion entre pratiques aboutissantes et fantomatiques. Mais tandis que dans les cas évoqués par Wittgenstein les usagers du langage croient, comme l'interlocuteur du (RP, $\mathbb{S} 414)$, qu'ils tissent une étoffe parce qu'ils sont assis devant un métier à tisser et qu'ils font les mouvements de tissage dans le vide ${ }^{2 \circ}$, Marx semble évoquer plutôt la situation inverse, où en achetant ou en échangeant des marchandises, on croit être en train de ne rien faire, alors que la transaction implique un travail réel, d'une part et de l'autre - le travail spécifique de tissage de l'étoffe, le travail spécifique qui produit la marchandise d'échange ou qui est à l'origine de l'argent de l'achat. La disparition des traces du travail spécifique, du contexte de production, et des liens de productions est bien entendu la plus flagrante lorsque les transactions se font avec de l'argent: «La marchandise disparaissant dans l'acte de sa conversion en argent, l'argent dont dispose un particulier ne laisse entrevoir ni comment il est tombé sous sa main ni quelle chose a été transformée en lui. Impossible de sentir, non olet, d'où il tire son origine» (c, p. 94).

Quand Marx dit que la marchandise «disparaît» dans sa conversion en argent, nous comprenons son expression à la fois de manière littérale - du point de vue du vendeur, la marchandise n'est plus là et à sa place, il y a de l'argent et figurée - avec l'objet marchandise disparaissent les dernières traces de ses différentes métamorphoses et des autres « disparitions » qu’elles entraînent, dont celle du travail et du travailleur qui est à son origine. C'est bien cette dernière « disparition » qu'il est question de donner à «voir », puisqu'elle n’est pas apparente, de manière à rétablir une relation sociale entre l'objet-marchandise et sa

19. La notion de travail chez Marx et Wittgenstein peut être mise en relation à celle du travail psychanalytique et du «travail du rêve », fondamentales dans la théorie psychanalytique. Voir à ce sujet, Sigmund Freud, L'interprétation des rêves [1900], Paris, Presses Universitaires de France, 2007. Cette question n’a pas pu être abordée ici, mais on peut rappeler que les modèles théoriques de Marx et Freud sont souvent rapprochés, tandis que Wittgenstein avait lu et commenté L'interprétation des rêves.

20. Au sujet de la confusion entre la signification du mot «soi » et l'état d'attention du philosophe qui veut en analyser la signification en se le répétant (RP, $\mathbb{\$} 413)$ : «Tu penses être obligé de tisser une étoffe, parce que tu es assis devant un métier à tisser - même s'il est vide - et parce que tu fais les mouvements du tissage ». (RP, $\mathbb{S} 414)$ 
valeur affichée. La difficulté bien sûr réside en ce qu'il est difficile de montrer ce qui n'est pas visible, notamment, les liens entre la marchandise et son prix ainsi que le travail (aussi social) sous-jacent à ces liens. Marx s'y prend, on le sait, en interposant entre la marchandise et le prix ainsi que les formes intermédiaires du produit et de la valeur fournissant ainsi une «genèse » de la forme monnaie ${ }^{21}$, en remontant jusqu'à ses sources dans le travail. Ces formes intermédiaires paradigmatiques (puisqu'elles n'illustrent pas un événement historique spécifique) permettent de «voir» le jeu d'apparitions et de disparitions des produits du travail et attirent notre attention sur les personnes physiques qui font le travail. Marx rappellera, par exemple, que malgré leur apparente autonomie, «[...] les marchandises ne peuvent point aller elles-mêmes au marché ni s'échanger ellesmêmes entre-elles. Il nous faut donc tourner notre regard vers leurs gardiens et conducteurs, c'est-à-dire vers leurs possesseurs.» (c, p. 77). Les différentes formes de la valeur renvoient ainsi aux différents rapports qu'entretiennent les gens entre eux et avec les choses: les possesseurs se croient possesseurs, mais se comportent à l'égard de leurs possessions comme des simples conducteurs et gardiens; et à l'égard d'autres possesseurs, comme des «ayants» de marchandise. De même qu'il y a lieu de distinguer entre une table-produit du travail et une tablemarchandise dansant sur sa tête, il y a lieu de remarquer que dans la mesure où les personnes se définissent par rapport aux produits du travail, ce n'est qu'en tant que travailleurs qu'elles maintiennent un lien actif avec ceux-ci, intervenant dans l'établissement de sa valeur par leur travail. Une fois que les acteurs agents se «transforment», eux, en possesseurs (ou conducteurs) de marchandises, ils perdent de vue leur rôle dans la détermination de la valeur des produits du travail et, avec lui, le sens de leur rôle social.

Wittgenstein insiste aussi sur l'importance de ce qu'il appelle les «maillons intermédiaires », c'est-à-dire des cas de figure qui donnent «à voir » des connexions entre les mots/formes d'expression et les jeux de langage/usages quotidiens, ainsi qu'entre les différentes formes d'expression entre elles:

Notre grammaire manque de caractère synoptique. La représentation synoptique nous procure la compréhension qui consiste à «voir des connexions». D’où l'importance qu'il y a à trouver et à inventer des maillons intermédiaires.

21. «Il s'agit maintenant [...] de fournir la genèse de la forme monnaie, c'est-à-dire de développer l'expression de la valeur contenue dans le rapport de valeur des marchandises depuis son ébauche la plus simple et la moins apparente jusqu’à cette forme monnaie qui saute aux yeux de tout le monde. En même temps sera résolue et disparaîtra l'énigme de la monnaie». (c, p. 50) 
Le concept de représentation synoptique a pour nous une signification fondamentale. Il désigne notre forme de représentation, la façon dont nous voyons les choses. (S'agit-il d'une «Weltanschaung»"22 ?) (RP, \122)

Dans les Recherches philosophiques, ces «maillons intermédiaires » sont des exemples d'usages de mots et d'expressions qui, en établissant un réseau de ressemblances et de différences, permettent de resituer le langage dans des pratiques spécifiques et de suivre les changements de sens que comportent les déplacements des mots d'un milieu discursif (jeu de langage) à un autre. Dans ce sens, Wittgenstein retrace les «métamorphoses » des mots, tout comme Marx reconstitue les changements de valeur des produits du travail, mettant autant en évidence ce que nous «voyons» dans l'usage des mots que ce qui nous échappe - disparaît - dans leur transfert d'une expression à une autre. Or, comme le fait Marx à travers sa genèse de l'argent, les «maillons intermédiaires » que Wittgenstein nous incite à «trouver ou à inventer » rappellent que, en dernière instance, c'est nous (usagers du langage) que nous ne voyons pas. Ainsi, Wittgenstein met souvent en scène les gestes corporels des interlocuteurs - leurs grimaces, leur manière de regarder au loin, sa propre manière de manifester physiquement un effort d'attention introspective ${ }^{23}$ - de manière à souligner, d'une part, le lien que chaque usager fait (fait le travail, agit) entre le sens et l'usage devient ainsi un médiateur actif dans la circulation sociale du sens, et de l'autre, l'écart que chaque usage établit entre les mots et le sens, figuré dans le même corps qui fait leur lien. Mais si les usagers du langage sont présentés, dans un sens, comme les «maillons intermédiaires » qui font «voir » le langage, ils ne sont pas pour autant des médiateurs improductifs, puisque ce sont eux qui mettent le langage au travail et qui le font produire. Lecteurs et interlocuteurs sont ainsi ramenés systématiquement à des considérations prosaïques - quels sont le but, le contexte, et l'utilité de leurs expressions? - qui les rappellent à leur propre activité sociale.

Dans les arguments de Marx et de Wittgenstein le registre visuel du disparaître, de l'apparition et de l'évidence signale un «point aveugle » de l'attention, ou une «faille» dans l'entendement, à l'égard des rapports sociaux et historiques qui

22. Les termes sont quelque peu différents dans la traduction anglaise de cette remarque, où au lieu du manque de caractère «synoptique» de la grammaire il s'agit de son manque de clarté (perspicuity).

23. «[...] Mais que peut bien signifier : «diriger mon attention sur ma conscience »? [...] je regardais fixement devant moi - mais aucun point ni aucun objet déterminés. J'écarquillais les yeux, mais ne fronçait pas les sourcils [...]. Mon regard était vacant ou analogue à celui d'un homme qui admire la lumière et s'abreuve de cette lumière. [...] » $\left(\mathrm{RP}, \mathbb{\int} 412\right)$ 
sous-tendent la perception. D’un point de vue très général, il exprime la difficulté méthodologique à saisir en même temps les éléments d'un ensemble en tant qu'éléments individuels et en tant que participants de cet ensemble - la difficulté à comprendre, dans le cas des rapports de production, la marchandise et sa valeur comme la «forme cellulaire économique » du «corps organisé » de l'économie et de la société (C, p. 35), ou bien, dans le cas de l'usage du langage, celle liée à l'effort d'appréhender les mots et les expressions de manière synoptique (RP, $\left.\int 122\right)$. Car, d'une part, ce sont les éléments isolés qui se donnent à la perception, mais d'autre part, leur évidence et leur autosuffisance occultent leur fonctionnement et leur valeur au sein de l'ensemble de pratiques où ils s'insèrent. Ainsi, toujours d'un point de vue méthodologique, Marx et Wittgenstein valorisent la figuration du type synechdocale des rapports qui les intéresse : chaque élément doit évoquer d'autres éléments (les «maillons intermédiaires » de Wittgenstein, les «formes » de Marx, qui sont autant de moments d'une métamorphose), et invoquer, par là même, les relations qui mettent en œuvre l'ensemble.

Il s'agit, en définitive, de développer une sorte de sensibilité ou d'attention aux productions humaines en tant que productions. Ici le registre de la disparition et de l'évidence met en relief une étrange, mais ancienne, contradiction: d'une part, nous «savons » que les produits - linguistiques et économiques - sont des produits sociaux et l'œuvre du travail humain, aussi ingérable soit-il ; d'autre part, en pratique, nous nous mettons, pour ainsi dire, à leur merci, ou du moins, nous nous laissons confondre par leur apparente autonomie et remettons en question notre propre contribution dans l'ensemble de la production. Encore une fois, ces doutes expriment un état de choses, à savoir, que les acteurs sociaux ne contrôlent pas l'ensemble de leurs productions, qu'ils n'agissent pas, d'ailleurs, d'une seule conscience, et qu'ils ne sont pas non plus les esclaves de leurs productions. Le registre de la disparition et de l'évidence sert à marquer, dans les arguments de Marx et de Wittgenstein, les moments où la balance du pouvoir bascule d'un côté ou de l'autre de cette ambivalence: les mots et les choses sont perçus comme maîtres, et la fonction des acteurs sociaux disparaît; ceux-ci règnent, et les mots et les choses, étrangement, perdent leur intelligibilité.

Mais si les acteurs sociaux ne sont pas maîtres de leurs rapports de production, comme l'aurait voulu Marx, ils en sont bien responsables, notamment par leur travail, comme le reconnaît aussi Wittgenstein. Le registre visuel qui nous intéresse devient alors plus littéral, puisqu'il sert à dénoter la difficulté à conceptualiser la part concrète des acteurs sociaux dans la production de la valeur et du sens, et l'absence ou la présence des corps physiques dans ces conceptualisations. Marx et Wittgenstein rappelleront ainsi la corporalité des acteurs à leurs 
lecteurs - les marchandises ne vont pas seules au marché, les formes d'expression comportent des gestes et des grimaces - sans toutefois centrer leur discours sur elle. «Tout signe isolé paraît mort», constate Wittgenstein, «[...] c'est dans l'usage qu'il est vivant» (RP, $\$ 432$ ); qui se demande ensuite: «A-t-il en lui-même le souffle de la vie? - ou l'usage est-il son souffle?» (RP, $\$ 432)$. Les signes et les choses, donc, sont animés; non pas directement par des corps humains, des individus spécifiques, mais par l'usage, qui les fait travailler. Ici nous sommes loin des registres de la domination et de la maîtrise pour exprimer le pouvoir social : les individus s'impliquent dans une activité, celle-ci anime la société. Or, cette activité n'est pas accessible sans porter l'attention aux corps qui la mettent en œuvre, au risque de transférer encore une fois le pouvoir d'animation à des instances inhumaines. D'où le glissement entre l'usage et les usagers, le travail et les travailleurs, dans les discours de nos deux philosophes, glissement qui, d'une part, ramène l'attention sur les travailleurs et usagers qui courent le danger de disparaître en étant assimilés à des simples moyens de production, et d'autre part, empêche l'attribution du pouvoir de production selon des schémas de domination et de maîtrise. Je pense qu'en dernière instance, c'est cette leçon qu'il importe de retenir du recours du registre de la disparition et de l'évidence tel qu'il fonctionne dans les arguments de Marx et de Wittgenstein : lorsque ce registre surgit, un déplacement dans l'attribution des pouvoirs est à l'œuvre, auquel il convient d'être attentif.

C'est bien cela qui préoccupait, d'ailleurs, l'amante du mage, dont le regard clairvoyant a inspiré ces considérations. Elle s'inquiétait, rappelons-le, du pouvoir de mystification du tour de disparition de son amant et des usages que d'autres pourraient en faire. Il se peut que ce soit le rôle d'amante qui, par le lien complexe et intime qu'il implique au corps et à la valeur de l'autre, et par l'approche à l'autre à la fois partielle et d'ensemble qu'il permet, lui ait donné la clarté de vision qui manquait à la foule. En tout cas, il semble qu'elle ait su distinguer, dans sa vision de la tour Eiffel, sa valeur d'usage (la tour touristique) de sa valeur d'échange (ce que la foule payait pour voir, c'est-à-dire, sa disparition), et qu'elle a réussi a garder en vue sa banalité physique, l'évidence qui, selon Wittgenstein, ne se voit plus, parce qu'elle est juste sous nos yeux - en dehors du champ visuel d'un télescope. 